\title{
Pain management strategies for patients on methadone maintenance therapy: a systematic review of the literature
}

\author{
Mel Clark Taveros, ${ }^{1}$ Elizabeth J Chuang ${ }^{2}$
}

- Additional material is published online only. To view please visit the journal online (http://dx.doi.org/10.1136/ bmjspcare-2016-001126).

${ }^{1}$ Hospice and Palliative Medicine, Montefiore Medical Center,

Bronx, New York, USA

${ }^{2}$ Department of Family and Social Medicine, Palliative Care Service, Montefiore Medical Center, Bronx, New York, USA

Correspondence to Dr Elizabeth J Chuang, Department of Family and Social Medicine, Palliative Care Service, Montefiore Medical Center, 3347 Steuben Avenue, 2nd Floor, Bronx, NY 10467-2490, USA; echuang@montefiore.org

This work was presented as a power-point presentation on 6 May 2015 at the New York City Fellows' Palliative Care Research Day.

Received 12 February 2016 Revised 18 July 2016

Accepted 7 August 2016 Published Online First 26 August 2016

\section{CrossMark}

To cite: Taveros MC, Chuang EJ. BMJ Supportive \& Palliative Care 2017;7:383-389.

\section{ABSTRACT}

Context Prescription opioid and heroin abuse has increased substantially in recent years.

Enrolment on opioid agonist therapy programmes is consequently increasing as well. As a result of these trends, more patients who present with acute pain secondary to a malignancy are also on chronic methadone maintenance therapy (MMT) for substance abuse. This combination of diagnoses presents a pain management challenge for palliative care providers.

Objectives This paper aims to gather and review the available medical literature pertaining to the use of opioid analgesia in methadonemaintained patients.

Methods The authors searched PubMED, PsychINFO, EMBASE, Clinical Key, the Cochrane Library and CINAHL from their inception to May 2015 for relevant articles.

All articles that discuss opioid therapy in adult patients on methadone maintenance for opioid addiction with cancer or advanced illness were included. Data were extracted and study quality was rated independently by the authors.

Results Our searches resulted in 680 hits. Of those, only 7 met inclusion criteria for the study. Most of the studies favoured the use of methadone either in scheduled divided doses every 4-8 hours or by continuous intravenous infusion. The overall strength of the evidence was poor, consisting mainly of case series, case reports and 1 single-centre retrospective cohort study lacking a comparison group.

Conclusions Treating MMT-maintained patients with methadone for analgesia may be preferable to using other opioid analgesics. However, there are many systems barriers that may make this approach challenging and the evidence favouring methadone over other opioid analgesics is weak.

\section{INTRODUCTION}

Palliative care teams are often consulted to assist in managing complex pain syndromes related to advanced disease and serious illnesses. More than half of patients with cancer develop pain, either from tumour burden, complications of advanced or progressive disease or from the effects of treatment regimens, including surgery, chemotherapy and radiotherapy. ${ }^{1}$

At the same time, the number of prescription opioid and heroin abusers is on the rise, ${ }^{2}$ and enrolment on opioid agonist therapy programmes is consequently increasing. As a result of these trends, there are more patients with pain secondary to a malignancy who are also on chronic methadone maintenance therapy (MMT) for substance abuse.

Multiple sources in the literature have shown that long-term use of opioids, including methadone, either illicitly or for therapeutic purposes, has profound effects on the perception of pain. ${ }^{3}$ The main effects include increased sensitivity to pain and decreased threshold of pain sensation, which together result in lowered pain tolerance. ${ }^{4}$ For patients on chronic MMT, effective acute pain management can be very difficult. In addition, patients with a history of substance abuse usually have comorbid chronic pain syndromes further complicating therapeutic management. ${ }^{5}$ Others may have continued illicit drug use and comorbid psychiatric disorders and a host of complex psychosocial issues that have been shown to affect perception of symptom burden and pain.

It is recognized that undertreatment of pain occurs relatively more frequently in patients with a history of chemical 
dependency, including patients on $\mathrm{MMT}^{6}$ Multiple barriers render effective acute pain management challenging. These may include patient-related factors such as under-reporting of pain due to fear of labelling of aberrant behaviour as 'drug seeking' and thereby withholding of medications by providers. ${ }^{7} 8$ Systems-related barriers include strict regulations and possible legal sanctions related to state-mandated opioid dispensing. ${ }^{8}$ Difficulties also arise from provider deficiencies in knowledge and skills in assessing and managing complicated pain exacerbated by fear of relapse. ${ }^{8}$ Pain in patients on long-term opioid agonist therapy requires careful attention to clinical and pharmacological principles for effective treatment. Misinformation may contribute to inadequate pain control.

Pain management and the avoidance of relapse of abuse are important outcomes for this patient population. In practice, physicians have used several approaches, including using opioids other than methadone for analgesia in addition to maintenance methadone, increasing the maintenance dose of methadone, adding methadone in divided doses to an unchanged methadone maintenance dose, and combining maintenance and analgesia dosing and dividing the total into several equal doses throughout the day. The paucity of evidence-based clinical guidelines in pain management specifically targeted for patients with concurrent MMT may contribute to the overall inefficiency and difficulty in achieving pain management and relapse avoidance. This paper aims to gather and review the available medical literature pertaining to opioid analgesic management strategies in methadonemaintained patients.

\section{METHODS}

\section{Data sources}

The Preferred Reporting Items for Systematic Reviews and Meta Analyses (PRISMA) statement was used to develop the methods for this systematic review. The authors searched PubMED, PsychINFO, EMBASE, Clinical Key, the Cochrane Library and CINAHL from their inception to May 2015. The following search terms were included: 'methadone maintenance' subheading 'therapy' and 'therapeutic use', 'acute pain', 'cancer pain', 'analgesia', 'addiction', 'addiction maintenance' subheading 'therapy', 'addictive behavior', 'opiate substitution treatment' and 'opioid agonist therapy'. MeSH terms were used when possible. An example of one complete search in PubMED is included in the online supplementary material. The reference lists of all included articles as well as several review articles on the topic were also hand-searched.

\section{Study selection}

We included all articles that discuss opioid therapy in adult patients on methadone maintenance for opioid addiction with cancer or advanced illness. We included clinical trials, cross-sectional observational studies, cohort studies, case-control studies, case series and case reports. No animal studies were included. Articles that focused on the use of methadone for pain management in patients without substance use history, articles on patients using buprenorphine maintenance therapy, articles on MMT rather than pain management, studies involving paediatric patients and articles that focus on perioperative pain or that focus on pregnant or postpartum women were all excluded. Articles without primary data such as review articles and clinical practice reviews were excluded, as well as pharmacokinetic articles. The search and review process were performed independently by the authors (MCT and EJC).

\section{Data extraction and quality assessment}

Data were extracted independently by the authors (MCT and EJC) using a standard data extraction form. Individual study quality was rated using the Strength of Recommendation Taxonomy (SORT) method for rating individual studies from level 1 (best quality evidence) to level 3 (poorest quality evidence). ${ }^{9}$ Quality of the body of evidence generated by all of the studies together was assessed using US Preventive Service Task Force criteria ratings of good, fair and poor. ${ }^{10}$ Disagreement between the authors was resolved with discussion.

\section{Data synthesis}

Results of individual studies and strengths and weaknesses of the body of evidence were described narratively. No statistical combination of results was possible, given that all but one study involved only case series or case studies.

\section{RESULTS}

Our searches resulted in 680 hits. Of those, only seven met inclusion criteria for the study. ${ }^{5}$ 11-16 Figure 1 shows the screening and selection process of the studies. Table 1 summarises the studies and their results. Most of the studies favoured the use of methadone for analgesia either in scheduled divided doses every 4-8 hours or by continuous infusion intravenously or subcutaneously. The overall strength of the evidence was poor, consisting of a retrospective, uncontrolled chart review, case series and case studies and only one case/control study with a comparison group that is not appropriate for the research question of this systematic review. Overall, the quality of evidence was poor.

\section{Participants}

The studies reported outcomes from a total of 142 participants, all adults over the age of 18 . There were 84 men and 58 women. Participants were studied 


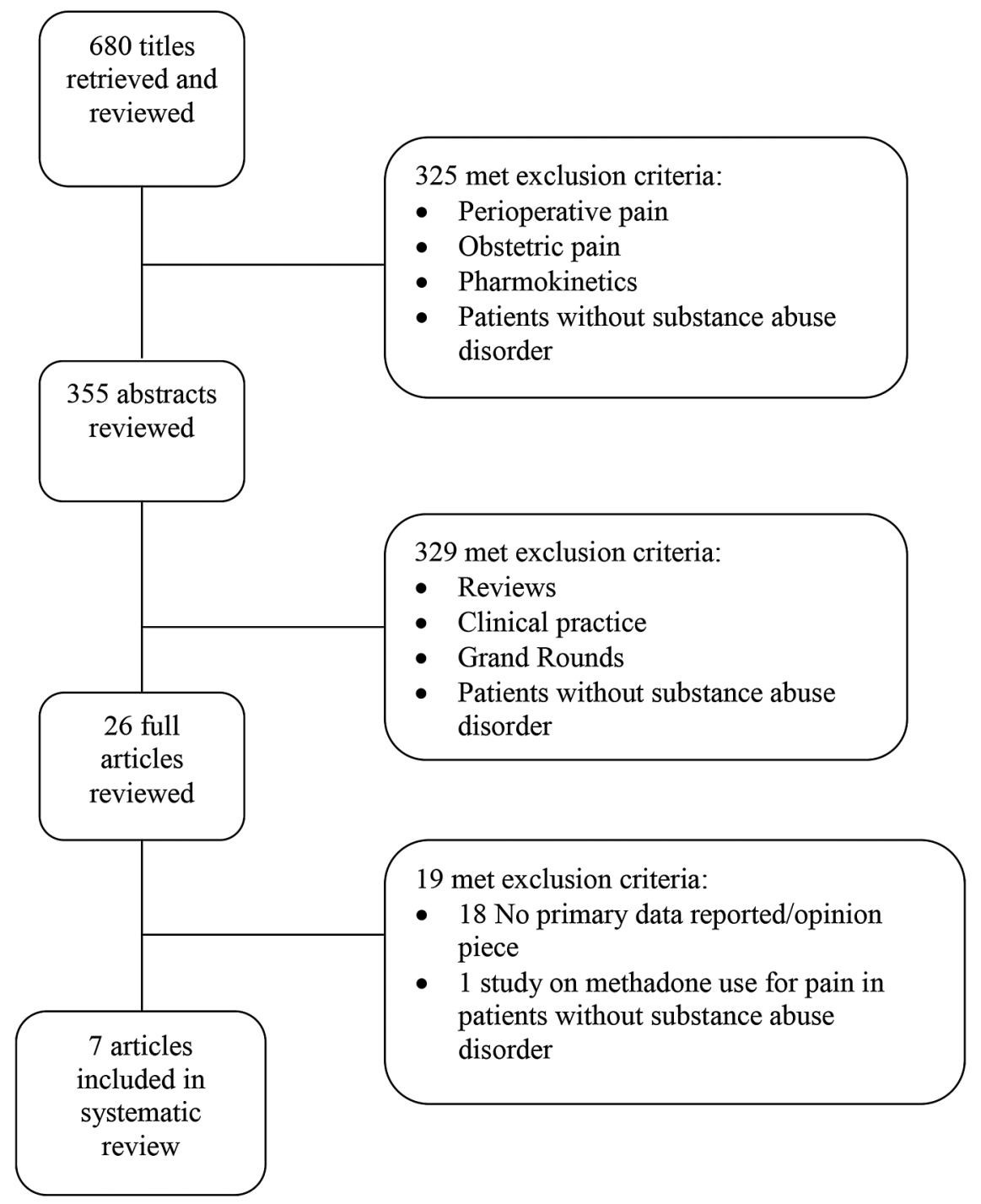

Figure 1 Flow diagram depicting selection of studies for inclusion in review.

from 1991 to 2013 and were from the USA, Australia, Germany and Ireland. All patients had a history of opioid abuse and almost all were receiving MMT treatment at the time of the study.

\section{Interventions}

Treatments were various and included increasing the maintenance dose of methadone, ${ }^{11}$ adding methadone in divided doses to an unchanged methadone maintenance dose, ${ }^{5}$ combining maintenance and analgesia dosing and dividing the total into several equal doses throughout the day, ${ }^{12}{ }^{13}$ use of continuous subcutaneous and intravenous methadone ${ }^{12}$ and use of opioids other than methadone for analgesia in addition to maintenance methadone. ${ }^{14}$ Oral methadone was given in one study, ${ }^{15}$ but the dosing schedule was not specified.

\section{Controls/comparisons}

Most studies lacked a comparison or control group. Some studies ${ }^{5} 1215$ reported pain control outcomes before and after treatment with methadone for analgesia, with the baseline pain measurement used as a comparison. Hines et $a l^{11}$ reported outcomes of a comparison group of patients not receiving MMT, but this comparison was not deemed relevant to the study question of this review.

\section{Risk of bias}

All of the studies reported here have a high risk of bias. Comparisons consisted mainly of preintervention and postintervention comparisons. Some studies reported only subjective outcomes such as 'good pain control' or 'poor pain control' or 'behavioural problems. ${ }^{13} 16$ Studies were observational; no patients were randomised to receive methadone or another opioid for analgesia. No studies included blinding of treatment groups for investigators, clinicians or patients. Generalisability of the results was low; samples were mainly single centre with few participants.

\section{Description of individual studies}

Blinderman et $a l^{5}$ performed a retrospective chart review of HIV-positive patients in a specialised pain 


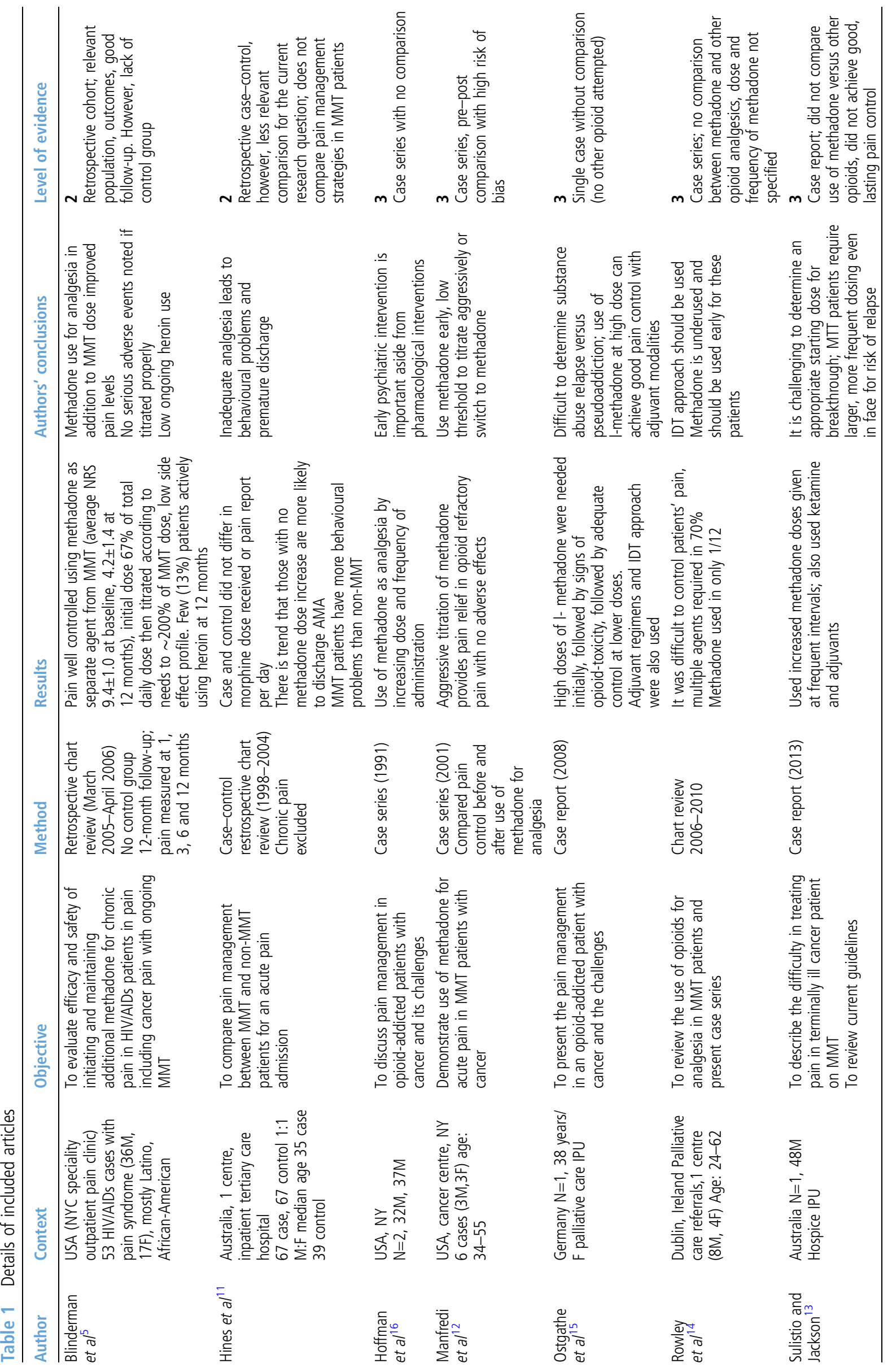


clinic in New York City from March 2005 to April 2006. They included 53 patients actively enrolled in methadone maintenance treatment programmes (MMTPs). They ranged from 18 and 75 years of age, $68 \%$ were men, $45 \%$ Latino and $45 \%$ AfricanAmerican. All were started on methadone for analgesia prescribed by the pain clinic, without a change in maintenance methadone dose provided by individual MMTPs. There was no comparison group. The average initial methadone maintenance dose was 102 $\pm 34 \mathrm{mg} /$ day. The dose of methadone for analgesia increase from about $2 / 3$ of the average maintenance dose $(61 \pm 34 \mathrm{mg} /$ day $)$ to about $200 \%$ of the average maintenance dose at the end of the study (200 $\pm 139 \mathrm{mg} /$ day). Pain was significantly reduced from the first visit $(9.4 \pm 1.0$ on NRS) to the last visit at 12 months (4.2 \pm 1.4 on NRS). At the end of the study, $13 \%$ of patients had urine toxicology studies positive for heroin. Side effects included constipation and fatigue in $6 \%$ of study participants, respectively.

Hines et $\mathrm{l}^{11}$ performed a retrospective case/control chart review of 67 patients on MMT admitted to the hospital for acute care from 1998. About half (50.7\%) were men and the average age was 35 . Controls were 67 patients not on MMT admitted to the hospital for acute care and matched to cases on age, gender and medical/surgical condition. The main outcome was median morphine equivalent dose of opioid use for analgesia, which was not significantly different between MMT patients $(5.07 \mathrm{mg} /$ day $)$ and controls $(6.67 \mathrm{mg} /$ day). MMT patients were significantly more likely to receive benzodiazepines and to have behavioural problems reported in charts. A minority of MTT patients (12\%) had their maintenance methadone dose increased during the hospitalisation. The authors report a non-significant correlation between lack of increase in methadone dose during admission and discharge against medical advice.

Hoffman et $\mathrm{l}^{16}$ present a case series of two patients in New York in 1991 with active or recent history of opioid abuse and painful complications of cancer. One patient was a man aged 32 years on MMT with colon cancer and pain from boney metastases. He had behavioural issues and non-compliance on both longacting morphine in addition to maintenance methadone and subsequently on methadone for analgesia (maintenance dose and analgesic dose combined and divided into equal doses every 6 hours) with hydromorphone for breakthrough pain. His behavioural issues resolved with the use of a pain contract. The second patient was a man aged 37 years with a history of heroin abuse, not on MMT, with pain related to likely epidural extension of large cell lymphoma. His pain was inadequately controlled and he had behavioural issues on hydromorphone $4 \mathrm{mg}$ every 4 hours as needed. These issues resolved when $20 \mathrm{mg}$ methadone four times a day was added to his regimen.
Manfredi et $a l^{12}$ report a case series of six patients with long-term exposure to methadone as treatment for heroin addiction treated at a cancer centre with methadone for cancer pain. They compare pain control before and after switching to methadone from other opioids for analgesia. Patients were 3 men and 3 women ranging in age from 34 to 55 . All patients achieved significant and lasting pain control with oral or intravenous methadone, five after failing initial treatment with one to two other opioids.

Ostgathe $e t a l^{15}$ reported in a letter to the editor a single case in 2008 of a woman aged 39 years on long-term MMT who developed pain related to cervical cancer. Her pain was controlled on very high doses of methadone, but she suffered symptoms of opioid overdose. Eventually, pain was controlled on lower doses of methadone.

Rowley et $a l^{14}$ conducted a retrospective chart review of patients being treated by a speciality palliative care service in Dublin between June 2006 and June 2010 who were on MMT. They identified eight men and four women ranging in age from 24 to 62 years with AIDS-related malignancy $(30 \%)$ and solid tumour malignancy (70\%). Seven patients were prescribed opioid analgesia, but only one was prescribed oral methadone as the opioid analgesic, and the dosing regimen was not specified. One additional patient received subcutaneous infusion of methadone at the time of death, but the rate was not specified. There was no comparison between methadone and other opioid use for analgesia on pain or other outcomes.

Finally, Sulistio and Jackson ${ }^{13}$ reported a single case of a man aged 48 years on MMT with severe pain related to metastatic malignancy of unknown origin in Australia in 2013. His pain was temporarily controlled with a change from once-daily methadone to twicedaily dosing along with 'burst' ketamine and dexamethasone. This article also focuses on the challenges of caring for a patient with social chaos at the end of life.

\section{DISCUSSION}

The aim of this study was to examine the available evidence on best practices for treating pain in patients on MMT for opioid addiction. Most of the studies were case series or case reports that were 'proof of concept' descriptions of the use of methadone as an analgesic in this population. Even within these reports, there were varying levels of success; Sulistio and Jackson ${ }^{13}$ report evidence of inadequate symptom control requiring the use of multiple adjuvants in addition to methadone and Ostgathe et $a l^{15}$ report varying success using methadone as an analgesic. The most successful case series come from Hoffman et $a l^{16}$ and Manfredi et $a l^{12}$ who describe good success in treating MMT-maintained patients with methadone as analgesia. 
The best overall evidence comes from Blinderman et $a l^{5}$ who presented a large cohort study documenting good efficacy using methadone as analgesia in addition to MMT in their patients. However, this study lacks a comparison group, making it high risk for potential bias. These results contrast with Rowley et al ${ }^{14}$ who report poor pain control in MMT patients treated with other opioids. The results of Hines et $a l^{11}$ are difficult to interpret in this setting; it appears that pain was not well controlled in the MMT patients in their cohort, and methadone was not specifically used for analgesia, although patients with increases in methadone maintenance doses appeared more satisfied with care, with a trend towards fewer discharges against medical advice.

Together, these studies are suggestive, although far from conclusive, that treating MMT-maintained patients with methadone for analgesia is an effective means of controlling pain and may be preferable to using other opioid analgesics. This is appealing on the one hand, given the simplicity of treating patients with one agent. However, methadone can prolong the Q-T interval with an associated increased risk of torsades de pointes with potentially fatal effect. Increasing the total daily dose of methadone for analgesic purposes must be done with caution and may warrant ECG monitoring. ${ }^{17}$ In addition, there are many systems barriers that may make this challenging. In the USA, and elsewhere, methadone prescribed for treatment of opioid addiction and methadone prescribed as analgesia are subject to different regulations. MMTPs and pain prescribers (whether palliative care practitioners, oncologists or primary providers) are often siloed. Practitioners without specialist training in palliative care may lack expertise in prescribing methadone for analgesia. This body of evidence does not make a strong case for abandoning the use of other long-acting or otherwise scheduled opioid analgesics as first line in this population. However, methadone for analgesia may become first line in the specialist palliative care setting for MMT-maintained patients.

These studies do highlight the sensitive and complicated nature of treating this population. Although non-opioid analgesics should be used as first line, many patients with serious or terminal illness will require opioid analgesia. Several concerns and difficulties may arise concerning the prescribing of opioids to these patients. The risk for relapse may be a concern for the patient and the clinician. Other potential problems like diversion and aberrant use of opioids are real. With any therapeutic relationship involving these controlled substances, there are safeguards that can be put in place prior to the undertaking of prescribing opioids to this subset of patients.

Regardless of opioid choice, strategies for optimal analgesia in patients on concurrent MMT include setting clear goals. The purpose of effective analgesia in the acute phase must clearly be set apart from preventing withdrawal symptoms and complications. This includes providing clear and exact instructions for doses and intervals and agents prescribed. Discussion of benefits and risks involved needs to take place and be documented in the chart accompanied by a signed patient-provider therapeutic contract. Honest communication of expectations is paramount. These expectations include that a single pharmacy will be used to fill prescriptions, that pill counting may be expected at certain times and that opioid prescriptions will be limited to a single provider.

Clinicians should start with a prescription of a limited supply of medication with the expectation of early follow-up. Patients should then be reassured that future refills should be determined by effective dose, patient adherence and nature of illness. It has to be clearly understood that if acute pain is expected to resolve or improve, opioids used for the purpose of analgesia will be tapered off. Communication and close coordination with the patient's methadone maintenance programme is also imperative. These patients are best served by an interdisciplinary approach to treatment of pain, physical and nonphysical, and appropriate referrals should be made to treat sources of pain. These referrals may include physical therapy, interventional pain and behavioural health.

This study also highlights a gap in the literature. Future studies should compare strategies for opioid analgesia, including starting with methadone for analgesia compared with initial treatment with other opioids, and within methadone treatment for analgesia comparing continuing methadone maintenance dose with the addition of scheduled doses for analgesia versus combining the maintenance and analgesic doses and dividing the total equally throughout the day. These, and many other questions regarding the optimal treatment of patients with opioid addiction near the end of life, remain unanswered.

Twitter Follow Elizabeth Chuang at @dr_liz_chuang

Acknowledgements The authors would like to thank Maria Elias, MLS, AHIP, for support in developing the search strategy. Competing interests None declared.

Provenance and peer review Not commissioned; externally peer reviewed.

\section{REFERENCES}

1 van den Beuken-van Everdingen MH, de Rijke JM, Kessels AG, et al. Prevalence of pain in patients with cancer: a systematic review of the past 40 years. Ann Oncol 2007;18:1437-49.

2 RuddRA, Aleshire, Zibbell JE, et al. Increases in drug and opioid overdose deaths-United States, 2000-2014. MMWR Morbid Mortal Wkly Rep 2016;64:1378-82.

3 Savage SR. Principles of pain treatment in the addicted patient. In: Graham AW, Schultz TK, eds. Principles of addiction medicine. 2nd edn. Chevy Chase, MD: American Society of Addiction Medicine, 1998:919-44. 
4 Huxtable CA, Roberts LJ, Somogyi AA, et al. Acute pain management in opioid-tolerant patients: a growing challenge. Anaesth Intensive Care 2011;39:804-23.

5 Blinderman CD, Sekine R, Zhang B, et al. Methadone as an analgesic for patients with chronic pain in methadone maintenance treatment programs (MMTPs). J Opioid Manag 2009;5:107-14.

6 Portenoy R, Dole V, Joseph H, et al. Pain management and chemical dependency. Evolving perspectives. JAMA 1997;278:592-3.

7 Doverty M, White JM, Somogyi AA, et al. Hyperalgesic responses in methadone maintenance patients. Pain 2001;90:91-6.

8 Scimeca MM, Savage SR, Portenoy R, et al. Treatment of pain in methadone-maintained patients. Mt Sinai J Med 2000;67:412-22.

9 Ebell MH, Siwek J, Weiss BD, et al. Strength of Recommendation Taxonomy (SORT): a patient-centered approach to grading evidence in the medical literature. Am Fam Physician 2004;69:548-56.

10 U.S. Preventive Services Task Force. Grade definitions. 2014. http://www.uspreventiveservicestaskforce.org/Page/Name/ grade-definitions (accessed 29 Oct 2015).
11 Hines S, Theodorou S, Williamson A, et al. Management of acute pain in methadone maintenance therapy in-patients. Drug Alcohol Rev 2008;27:519-23.

12 Manfredi PL, Gonzales GR, Cheville AL, et al. Methadone analgesia in cancer pain patients on chronic methadone maintenance therapy. J Pain Symptom Manage 2001;21: 169-74.

13 Sulistio M, Jackson K. Three weeks from diagnosis to death: the chaotic journey of a long-term methadone maintenance patient with terminal cancer. J Pain Symptom Manage 2013;46 (4):598-602.

14 Rowley D, McLean S, O'Gorman A, et al. Review of cancer pain management in patients receiving maintenance methadone therapy. Am J Hosp Palliat Care 2011;28:183-7.

15 Ostgathe C, Gaertner J, Nauck F, et al. High dose levomethadone treatment for cancer pain in a patient with a history of drug addiction (letter). J Pain Symptom Manage 2008;35:229-30.

16 Hoffman M, Provatas A, Lyver A, et al. Pain management in the opioid-addicted patient with cancer. Cancer 1991;68:1121-2.

17 Stringer J, Welsh C, Tommasello A. Methadone-associated Q-T interval prolongation and torsades de pointes. Am J Health Syst Pharm 2009;66:825-33. 